\title{
Degree distribution of an inhomogeneous random intersection graph
}

\author{
Mindaugas Bloznelis* \\ Faculty of mathematics and informatics \\ Vilnius University \\ Vilnius, Lithuania \\ mindaugas.bloznelis@mif.vu.lt
}

\author{
Julius Damarackas \\ Faculty of mathematics and informatics \\ Vilnius University \\ Vilnius, Lithuania \\ julius.damarackas@mif.stud.vu.It
}

Submitted: Oct 8, 2012; Accepted: Jul 11, 2013; Published: Jul 19, 2013

Mathematics Subject Classifications: 05C80, 05C07, 05C82

\begin{abstract}
We show the asymptotic degree distribution of the typical vertex of a sparse inhomogeneous random intersection graph.
\end{abstract}

Keywords: degree distribution; random graph; random intersection graph; power law

\section{Introduction}

Let $X_{1}, \ldots, X_{m}, Y_{1}, \ldots, Y_{n}$ be independent non-negative random variables such that each $X_{i}$ has the probability distribution $P_{1}$ and each $Y_{j}$ has the probability distribution $P_{2}$. Given realized values $X=\left\{X_{i}\right\}_{i=1}^{m}$ and $Y=\left\{Y_{j}\right\}_{j=1}^{n}$ we define the random bipartite graph $H_{X, Y}$ with the bipartition $V=\left\{v_{1}, \ldots, v_{n}\right\}, W=\left\{w_{1}, \ldots, w_{m}\right\}$, where edges $\left\{w_{i}, v_{j}\right\}$ are inserted with probabilities $p_{i j}=\min \left\{1, X_{i} Y_{j}(n m)^{-1 / 2}\right\}$ independently for each $\{i, j\} \in[m] \times[n]$. The inhomogeneous random intersection graph $G\left(P_{1}, P_{2}, n, m\right)$ defines the adjacency relation on the vertex set $V$ : vertices $v^{\prime}, v^{\prime \prime} \in V$ are declared adjacent (denoted $v^{\prime} \sim v^{\prime \prime}$ ) whenever $v^{\prime}$ and $v^{\prime \prime}$ have a common neighbour in $H_{X, Y}$.

The degree distribution of the typical vertex of the random graph $G\left(P_{1}, P_{2}, n, m\right)$ has been first considered by Shang [21]. The proof of the main result of [21] contains gaps and the result is incorrect in the regime where $m / n \rightarrow \beta \in(0,+\infty)$ as $m, n \rightarrow+\infty$. We remark that this regime is of particular importance, because it leads to inhomogeneous graphs with the clustering property: the clustering coefficient $\mathbf{P}\left(v_{1} \sim v_{2} \mid v_{1} \sim v_{3}, v_{2} \sim v_{3}\right)$ is bounded away from zero provided that $0<\beta<+\infty$ and $\mathbf{E} X_{1}^{3}<\infty$, $\mathbf{E} Y_{1}^{2}<\infty$, see [8].

${ }^{*}$ Supported by the Research Council of Lithuania Grant MIP-053/2011. 
The aim of the present paper is to show the asymptotic degree distribution in the case where $m / n \rightarrow \beta$ for some $\beta \in[0,+\infty]$.

We consider a sequence of graphs $\left\{G_{n}=G\left(P_{1}, P_{2}, n, m\right)\right\}$, where $m=m_{n} \rightarrow+\infty$ as $n \rightarrow \infty$, and where $P_{1}, P_{2}$ do not depend on $n$. We denote $a_{k}=\mathbf{E} X_{1}^{k}, b_{k}=\mathbf{E} Y_{1}^{k}$. By $d(v)=d_{G_{n}}(v)$ we denote the degree of a vertex $v$ in $G_{n}$ (the number of vertices adjacent to $v$ in $\left.G_{n}\right)$. We remark that for every $n$ the random variables $d_{G_{n}}\left(v_{1}\right), \ldots, d_{G_{n}}\left(v_{n}\right)$ are identically distributed. In Theorem 1 below we show the asymptotic distribution of $d\left(v_{1}\right)$.

Theorem 1. Let $m, n \rightarrow \infty$.

(i) Assume that $m / n \rightarrow 0$. Suppose that that $\mathbf{E} X_{1}<\infty$. Then $\mathbf{P}\left(d\left(v_{1}\right)=0\right)=1-o(1)$.

(ii) Assume that $m / n \rightarrow \beta$ for some $\beta \in(0,+\infty)$. Suppose that $\mathbf{E} X_{1}^{2}<\infty$ and $\mathbf{E} Y_{1}<\infty$. Then $d\left(v_{1}\right)$ converges in distribution to the random variable

$$
d_{*}=\sum_{j=1}^{\Lambda_{1}} \tau_{j},
$$

where $\tau_{1}, \tau_{2}, \ldots$ are independent and identically distributed random variables independent of the random variable $\Lambda_{1}$. They are distributed as follows. For $r=0,1,2, \ldots$, we have

$$
\mathbf{P}\left(\tau_{1}=r\right)=\frac{r+1}{\mathbf{E} \Lambda_{2}} \mathbf{P}\left(\Lambda_{2}=r+1\right) \quad \text { and } \quad \mathbf{P}\left(\Lambda_{i}=r\right)=\mathbf{E} e^{-\lambda_{i}} \frac{\lambda_{i}^{r}}{r !}, \quad i=1,2 .
$$

Here $\lambda_{1}=Y_{1} a_{1} \beta^{1 / 2}$ and $\lambda_{2}=X_{1} b_{1} \beta^{-1 / 2}$.

(iii) Assume that $m / n \rightarrow+\infty$. Suppose that $\mathbf{E} X_{1}^{2}<\infty$ and $\mathbf{E} Y_{1}<\infty$. Then $d\left(v_{1}\right)$ converges in distribution to a random variable $\Lambda_{3}$ having the probability distribution

$$
\mathbf{P}\left(\Lambda_{3}=r\right)=\mathbf{E} e^{-\lambda_{3}} \frac{\lambda_{3}^{r}}{r !}, \quad r=0,1, \ldots
$$

Here $\lambda_{3}=Y_{1} a_{2} b_{1}$.

Remark 2. The probability distributions $P_{\Lambda_{i}}$ of $\Lambda_{i}, i=1,2,3$, are Poisson mixtures. One way to sample from the distribution $P_{\Lambda_{i}}$ is to generate random variable $\lambda_{i}$ and then, given $\lambda_{i}$, to generate Poisson random variable with the parameter $\lambda_{i}$. The realized value of the Poisson random variable has the distribution $P_{\Lambda_{i}}$.

Remark 3. The asymptotic distributions (1) and (3) admit heavy tails. In the case (ii) we obtain a power law asymptotic degree distribution (1) provided that the heavier of the tails $t \rightarrow \mathbf{P}\left(\tau_{1}>t\right)$ and $t \rightarrow \mathbf{P}\left(\Lambda_{1}>t\right)$ has a power law, see, e.g., [13]. Similarly, in the case (iii) we obtain a power law asymptotic degree distribution (3) provided that $P_{2}$ has a power law.

Remark 4. Since the second moment $a_{2}$ does not show up in (1), (2) we expect that in the case (ii) the second moment condition $\mathbf{E} X_{1}^{2}<\infty$ is redundant and could perhaps be replaced by the weaker first moment condition $\mathbf{E} X_{1}<\infty$. 
Random intersection graphs have attracted considerable attention in the recent literature, see, e.g., [1, 2, 3, 9, 10, 12, 19]. Starting with the paper by Karoński et al [17], see also [22], where the case of degenerate distributions $P_{1}=P_{1 n}, P_{2}=P_{2 n}$ depending on $n$ was considered (i.e., $\mathbf{P}_{1 n}\left(c_{n}\right)=\mathbf{P}_{2 n}\left(c_{n}\right)=1$, for some $c_{n}>0$ ), several more complex random intersection graph models were later introduced by Godehardt and Jaworski [14], Spirakis et al. [18], Shang [21]. The asymptotic degree distribution for various random intersection graph models was shown in $[4,5,6,7,11,15,16,20,23]$.

\section{Proof}

Before the proof of Theorem 1 we introduce some notation and give two auxiliary lemmas.

The event that the edge $\left\{w_{i}, v_{j}\right\}$ is present in $H=H_{X, Y}$ is denoted $w_{i} \rightarrow v_{j}$. We denote

$$
\mathbb{I}_{i j}=\mathbb{I}_{\left\{w_{i} \rightarrow v_{j}\right\}}, \quad \mathbb{I}_{i}=\mathbb{I}_{i 1}, \quad u_{i}=\sum_{2 \leqslant j \leqslant n} \mathbb{I}_{i j}, \quad L=\sum_{i=1}^{m} u_{i} \mathbb{I}_{i}
$$

and remark that $u_{i}$ counts all neighbours of $w_{i}$ in $H$ belonging to the set $V \backslash\left\{v_{1}\right\}$. Denote

$$
\begin{aligned}
& \hat{a}_{k}=m^{-1} \sum_{1 \leqslant i \leqslant m} X_{i}^{k}, \quad \hat{b}_{k}=n^{-1} \sum_{2 \leqslant j \leqslant n} Y_{j}^{k}, \\
& \lambda_{i j}=\frac{X_{i} Y_{j}}{\sqrt{m n}}, \quad S_{X Y}=\sum_{i=1}^{m} \sum_{j=2}^{n} \frac{X_{i}}{\sqrt{m n}} \lambda_{i j} \min \left\{1, \lambda_{i j}\right\},
\end{aligned}
$$

and introduce the event $\mathcal{A}_{1}=\left\{\lambda_{i 1}<1,1 \leqslant i \leqslant m\right\}$. We denote $\beta_{n}=m / n$. By $\mathbf{P}_{1}$ and $\mathbf{E}_{1}$ we denote the conditional probability and conditional expectation given $Y_{1}$. By $\tilde{\mathbf{P}}$ and $\tilde{\mathbf{E}}$ we denote the conditional probability and conditional expectation given $X, Y$. By $d_{T V}(\zeta, \xi)$ we denote the total variantion distance between the probability distributions of random variables $\zeta$ and $\xi$. In the case where $\zeta, \xi, X, Y$ are defined on the same probability space we denote by $\tilde{d}_{T V}(\zeta, \xi)$ the total variation distance between the conditional distributions of $\zeta$ and $\xi$ given $X, Y$.

In the proof below we use the following simple fact about the convergence of a sequence of random variables $\left\{\varkappa_{n}\right\}$ :

$$
\varkappa_{n}=o_{P}(1), \quad \mathbf{E} \sup _{n} \varkappa_{n}<\infty \Rightarrow \mathbf{E} \varkappa_{n}=o(1) .
$$

We remark that the condition $\mathbf{E}_{\sup _{n}} \varkappa_{n}<\infty$ (which assumes implicitly that all $\varkappa_{n}$ are defined on the same probability space) can be replaced by a more restrictive condition that there exists a constant $c>0$ such that $\left|\varkappa_{n}\right| \leqslant c$, for all $n$. In the latter case we do not need all $\varkappa_{n}$ to be defined on the same probability space. In particular, given a sequence of bivariate random vectors $\left\{\left(\eta_{n}, \theta_{n}\right)\right\}$ such that, for every $n$ and $m=m_{n}$ random variables $\eta_{n}, \theta_{n}$ and $\left\{X_{i}\right\}_{i=1}^{m},\left\{Y_{j}\right\}_{j=1}^{n}$ are defined on the same probability space, we have

$$
\tilde{d}_{T V}\left(\eta_{n}, \theta_{n}\right)=o_{P}(1) \Rightarrow d_{T V}\left(\eta_{n}, \theta_{n}\right) \leqslant \mathbf{E} \tilde{d}_{T V}\left(\eta_{n}, \theta_{n}\right)=o(1) .
$$

Here and below all limits are taken as $n \rightarrow+\infty$ (if not stated otherwise). 
Lemma 5. Assume that $\mathbf{E} X_{1}^{2}<\infty$ and $\mathbf{E} Y_{1}<\infty$. We have as $n \rightarrow+\infty$

$$
\begin{aligned}
& \mathbf{P}\left(d\left(v_{1}\right) \neq L\right)=o(1), \\
& \mathbf{P}\left(\mathcal{A}_{1}\right)=1-o(1), \\
& S_{X Y}=o_{P}(1), \quad \mathbf{E} S_{X Y}=o(1) .
\end{aligned}
$$

Proof. Proof of (6). We observe that the event $\mathcal{A}=\left\{d\left(v_{1}\right) \neq L\right\}$ occurs in the case where for some $2 \leqslant j \leqslant n$ and some distinct $i_{1}, i_{2} \in[m]$ the event $\mathcal{A}_{i_{1}, i_{2}, j}=\left\{w_{i_{1}} \rightarrow\right.$ $\left.v_{1}, w_{i_{1}} \rightarrow v_{j}, w_{i_{2}} \rightarrow v_{1}, w_{i_{2}} \rightarrow v_{j}\right\}$ occurs. From the union bound and the inequality $\tilde{\mathbf{P}}\left(\mathcal{A}_{i_{1}, i_{2}, j}\right) \leqslant m^{-2} n^{-2} X_{i_{1}}^{2} X_{i_{2}}^{2} Y_{1}^{2} Y_{j}^{2}$ we obtain

$$
\tilde{\mathbf{P}}(\mathcal{A})=\tilde{\mathbf{P}}\left(\bigcup_{\left\{i_{1}, i_{2}\right\} \subset[m]} \bigcup_{2 \leqslant j \leqslant n} \mathcal{A}_{i_{1}, i_{2}, j}\right) \leqslant n^{-1} \hat{b}_{2} Y_{1}^{2} Q_{X} .
$$

Here $Q_{X}=m^{-2} \sum_{\left\{i_{1}, i_{2}\right\} \subset[m]} X_{i_{1}}^{2} X_{i_{2}}^{2}$. We note that $Q_{X}$ and $Y_{1}^{2}$ are stochastically bounded and $n^{-1} \hat{b}_{2}=o_{P}(1)$ as $n \rightarrow+\infty$. Therefore, $\tilde{\mathbf{P}}(\mathcal{A})=o_{P}(1)$. Now (5) implies (6).

Proof of (7). Let $\overline{\mathcal{A}}_{1}$ denote the complement event to $\mathcal{A}_{1}$. We have, by the union bound and Markov's inequality,

$$
\mathbf{P}_{1}\left(\overline{\mathcal{A}}_{1}\right) \leqslant \sum_{i \in[m]} \mathbf{P}_{1}\left(\lambda_{i 1} \geqslant 1\right) \leqslant(n m)^{-1} Y_{1}^{2} \sum_{i \in[m]} \mathbf{E} X_{i}^{2}=n^{-1} a_{2} Y_{1}^{2} .
$$

We conclude that $\mathbf{P}_{1}\left(\overline{\mathcal{A}}_{1}\right)=o_{P}(1)$, and now (5) implies $\mathbf{P}\left(\overline{\mathcal{A}}_{1}\right)=\mathbf{E P}_{1}\left(\overline{\mathcal{A}}_{1}\right)=o(1)$.

Proof of (8). Since the first bound of (8) follows from the second one, we only prove the latter. Denote $\hat{X}_{1}=\max \left\{X_{1}, 1\right\}$ and $\hat{Y}_{1}=\max \left\{Y_{1}, 1\right\}$. We observe that $\mathbf{E} X_{1}^{2}<\infty$, $\mathbf{E} Y_{1}<\infty$ implies

$$
\lim _{t \rightarrow+\infty} \mathbf{E} \hat{X}_{1}^{2} \mathbb{I}_{\left\{\hat{X}_{1}>t\right\}}=0, \quad \lim _{t \rightarrow+\infty} \mathbf{E} \hat{Y}_{1} \mathbb{I}_{\left\{\hat{Y}_{1}>t\right\}}=0 .
$$

Hence one can find a strictly increasing function $\varphi:[1,+\infty) \rightarrow[0,+\infty)$ with $\lim _{t \rightarrow+\infty} \varphi(t)$ $=+\infty$ such that

$$
\mathbf{E} \hat{X}_{1}^{2} \varphi\left(\hat{X}_{1}\right)<\infty, \quad \mathbf{E} \hat{Y}_{1} \varphi\left(\hat{Y}_{1}\right)<\infty .
$$

We remark that one can find $\varphi$ which satisfies, in addition, the inequalities

$$
\varphi(t)<t \quad \text { and } \quad \varphi(s t) \leqslant \varphi(t) \varphi(s), \quad \forall s, t \geqslant 1 .
$$

For this purpose we choose $\varphi$ of the form $\varphi(t)=e^{\psi(\ln (t))}$, where $\psi:[0,+\infty) \rightarrow[0,+\infty)$ is a differentiable concave function, which grows slowly enough to satisfy (10) and the first inequality of (11), and takes value 0 at the origin. We note that the second inequality of (11) follows from the concavity property of $\psi$. We remark, that (11) implies

$$
t /(s t)=s^{-1} \leqslant 1 / \varphi(s) \leqslant \varphi(t) / \varphi(s t), \quad s, t \geqslant 1 .
$$


Let $\hat{S}_{X Y}$ be defined as in (4) above, but with $\lambda_{i j}$ replaced by $\hat{\lambda}_{i j}=\hat{X}_{i} \hat{Y}_{j} / \sqrt{m n}$. We note, that $S_{X Y} \leqslant \hat{S}_{X Y}$. Furthermore, from the inequalities

$$
\min \left\{1, \hat{\lambda}_{i j}\right\} \leqslant \min \left\{1, \frac{\varphi\left(\hat{X}_{i} \hat{Y}_{j}\right)}{\varphi(\sqrt{m n})}\right\} \leqslant \frac{\varphi\left(\hat{X}_{i} \hat{Y}_{j}\right)}{\varphi(\sqrt{m n})} \leqslant \frac{\varphi\left(\hat{X}_{i}\right) \varphi\left(\hat{Y}_{j}\right)}{\varphi(\sqrt{m n})}
$$

we obtain $S_{X Y} \leqslant \hat{S}_{X Y} \leqslant S_{X Y}^{*} / \varphi(\sqrt{n m})$, where

$$
S_{X Y}^{*}=(m n)^{-1}\left(\sum_{1 \leqslant i \leqslant m} \hat{X}_{i}^{2} \varphi\left(\hat{X}_{i}\right)\right)\left(\sum_{2 \leqslant j \leqslant n} \hat{Y}_{j} \varphi\left(\hat{Y}_{j}\right)\right)
$$

We remark that (11) and (12) and imply the third and the first inequality of (13), respectively.

Finally, the bound $\mathbf{E} S_{X Y}=o(1)$ follows from the inequality $S_{X Y} \leqslant S_{X Y}^{*} / \varphi(\sqrt{n m})$ and the fact that $\mathbf{E} S_{X Y}^{*}$ remains bounded as $n, m \rightarrow+\infty$, see (10).

In the proof of Theorem 1 we use the following inequality referred to as LeCam's inequality, see e.g., [24].

Lemma 6. Let $S=\mathbb{I}_{1}+\mathbb{I}_{2}+\cdots+\mathbb{I}_{n}$ be the sum of independent random indicators with probabilities $\mathbf{P}\left(\mathbb{I}_{i}=1\right)=p_{i}$. Let $\Lambda$ be Poisson random variable with mean $p_{1}+\cdots+p_{n}$. The total variation distance between the distributions $P_{S}$ of $P_{\Lambda}$ of $S$ and $\Lambda$

$$
\sup _{A \subset\{0,1,2 \ldots\}}|\mathbf{P}(S \in A)-\mathbf{P}(\Lambda \in A)|=\frac{1}{2} \sum_{k \geqslant 0}|\mathbf{P}(S=k)-\mathbf{P}(\Lambda=k)| \leqslant \sum_{1 \leqslant i \leqslant n} p_{i}^{2} .
$$

Proof of Theorem 1. The case (i). Since $\mathbf{P}\left(d\left(v_{1}\right) \leqslant L\right)=1$ it suffices to show that $\mathbf{P}(L>\varepsilon)=o(1)$, for any $\varepsilon \in(0,1)$. For this purpose we write $\mathbf{P}(L>\varepsilon)=\mathbf{E P}_{1}(L>\varepsilon)$ and prove that $\mathbf{P}_{1}(L>\varepsilon)=o_{P}(1)$, see (5). Here we estimate $\mathbf{P}_{1}(L>\varepsilon)$ using the union bound and Markov's inequality,

$$
\mathbf{P}_{1}(L>\varepsilon) \leqslant \sum_{1 \leqslant k \leqslant m} \mathbf{P}_{1}\left(\mathbb{I}_{k}=1\right) \leqslant \mathbf{E}_{1} \sum_{1 \leqslant k \leqslant m} \lambda_{k 1}=\sqrt{m / n} Y_{1} \mathbf{E} X_{1}=o_{P}(1) .
$$

The case (ii). Before the proof we introduce some notation. Given $X, Y$, let

$$
\left\{\Delta_{l k}\right\}_{k=1}^{m}, \quad l=1,2,3, \quad\left\{\xi_{r k}\right\}_{k=1}^{m}, \quad r=1,2,3,4, \quad \text { and } \quad\left\{\xi_{3 k}^{\prime}\right\}_{k=1}^{m}, \quad\left\{\xi_{4 k}^{\prime}\right\}_{k=1}^{m},
$$

and $\left\{\eta_{k}\right\}_{k=1}^{m}$ be sequences of conditionally independent (within each sequence) Poisson random variables with mean values

$$
\begin{aligned}
& \tilde{\mathbf{E}} \xi_{1 k}=\sum_{2 \leqslant j \leqslant n} p_{k j}, \quad \tilde{\mathbf{E}} \xi_{2 k}=\sum_{2 \leqslant j \leqslant n} \lambda_{k j}, \quad \tilde{\mathbf{E}} \xi_{3 k}=\frac{1}{\sqrt{\beta_{n}}} b_{1} X_{k}, \quad \tilde{\mathbf{E}} \xi_{4 k}=\frac{1}{\sqrt{\beta}} b_{1} X_{k}, \\
& \tilde{\mathbf{E}} \Delta_{1 k}=\sum_{2 \leqslant j \leqslant n}\left(\lambda_{k j}-p_{k j}\right), \quad \tilde{\mathbf{E}} \Delta_{2 k}=\frac{1}{\sqrt{\beta_{n}}} \delta_{2} X_{k}, \quad \tilde{\mathbf{E}} \Delta_{3 k}=\frac{1}{\sqrt{\beta_{n}}} \delta_{3} X_{k}, \\
& \tilde{\mathbf{E}} \xi_{3 k}^{\prime}=\beta_{n}^{-1 / 2} b X_{k}, \quad \tilde{\mathbf{E}} \xi_{4 k}^{\prime}=\beta^{\prime} b_{1} X_{k}, \quad \tilde{\mathbf{E}} \eta_{k}=\lambda_{k 1} .
\end{aligned}
$$


Here we denote $b:=\min \left\{\hat{b}_{1}, b_{1}\right\}, \delta_{2}:=\hat{b}_{1}-b, \delta_{3}:=b_{1}-b$ and $\beta^{\prime}:=\min \left\{\beta^{-1 / 2}, \beta_{n}^{-1 / 2}\right\}$. We recall that $\tilde{\mathbf{E}}$ denotes the conditional expectation given $X, Y$. We assume that, given $X, Y$, the sequences $\left\{\xi_{3 k}^{\prime}\right\}_{k=1}^{m},\left\{\Delta_{2 k}\right\}_{k=1}^{m},\left\{\Delta_{3 k}\right\}_{k=1}^{m}$ are conditionally independent, the sequences $\left\{\mathbb{I}_{k}\right\}_{k=1}^{m},\left\{\xi_{3 k}\right\}_{k=1}^{m}$ are conditionally independent, the sequences $\left\{\xi_{1 k}\right\}_{k=1}^{m},\left\{\Delta_{1 k}\right\}_{k=1}^{m}$ are conditionally independent, and the sequence $\left\{\eta_{k}\right\}_{k=1}^{m}$ is conditionally independent of the sequences (15). We assume, in addition, that for $1 \leqslant k \leqslant m$

$$
\xi_{2 k}:=\xi_{1 k}+\Delta_{1 k}, \quad \xi_{3 k}:=\xi_{4 k}^{\prime}+\left(\beta_{n}^{-1 / 2}-\beta^{\prime}\right) b_{1} X_{k}, \quad \xi_{4 k}:=\xi_{4 k}^{\prime}+\left(\beta^{-1 / 2}-\beta^{\prime}\right) b_{1} X_{k} .
$$

Furthermore, we suppose that, given $X, Y_{1}$, the sequence $\left\{\eta_{k}\right\}_{k=1}^{m}$ is conditionally independent of the subgraph of $H_{X, Y}$ spanned by the vertices $\left\{v_{2}, \ldots, v_{n}\right\} \cup W$.

Next we introduce several random variables which are intermediate between $L$ and $d_{*}$ :

$$
\begin{aligned}
& L_{0}=\sum_{1 \leqslant k \leqslant m} \eta_{k} u_{k}, \quad L_{r}=\sum_{1 \leqslant k \leqslant m} \eta_{k} \xi_{r k}, \quad r=1,2,3,4, \\
& L_{2}^{\prime}=\sum_{1 \leqslant k \leqslant m} \eta_{k}\left(\xi_{3 k}^{\prime}+\Delta_{2 k}\right), \quad L_{3}^{\prime}=\sum_{1 \leqslant k \leqslant m} \eta_{k}\left(\xi_{3 k}^{\prime}+\Delta_{3 k}\right) .
\end{aligned}
$$

Finally, we denote

$$
\begin{aligned}
& f_{\tau}(t)=\mathbf{E} e^{i t \tau_{1}}, \quad \bar{f}_{\tau}(t)=\sum_{r \geqslant 0} e^{i t r} \bar{p}_{r}, \quad \bar{p}_{r}=\bar{\lambda}^{-1} \sum_{1 \leqslant k \leqslant m} \lambda_{k 1} \mathbb{I}_{\left\{\xi_{4 k}=r\right\}}, \quad \bar{\lambda}=\sum_{k=1}^{m} \lambda_{k 1}, \\
& \delta=\left(\bar{f}_{\tau}(t)-1\right) \bar{\lambda}-\left(f_{\tau}(t)-1\right) \lambda_{1}, \quad f(t)=\mathbf{E}_{1} e^{i t d_{*}}, \quad \bar{f}(t)=\overline{\mathbf{E}} e^{i t L_{4}} .
\end{aligned}
$$

Here $\overline{\mathbf{E}}$ denotes the conditional expectation given $X, Y$ and $\xi_{41}, \ldots, \xi_{4 m}$. We recall that $\mathbf{E}_{1}$ denotes the conditional expectation given $Y_{1}$.

Let us prove the statement (ii). In view of (6) the random variables $d\left(v_{1}\right)$ and $L$ have the same asymptotic distribution (if any). We shall show that $L$ converges in distribution to (1). We proceed in two steps. Firstly, we approximate the distribution of $L$ by that of $L_{4}$ using LeCam's inequality, see Lemma 6, and a coupling argument. Secondly, we prove the convergence of the characteristic functions $\mathbf{E} e^{i t L_{4}} \rightarrow \mathbf{E} e^{i t d_{*}}$. Here and below $i=\sqrt{-1}$ denotes the imaginary unit.

Step 1. Let us show that $L$ and $L_{4}$ have the same asymptotic probability distribution (if any). To this aim we prove that

$$
\begin{array}{ll}
d_{T V}\left(L, L_{0}\right)=o(1), & d_{T V}\left(L_{0}, L_{1}\right)=o(1), \\
\mathbf{E}\left|L_{1}-L_{2}\right|=o(1), & L_{2}^{\prime}-L_{3}^{\prime}=o_{P}(1), \quad \mathbf{E}\left|L_{3}-L_{4}\right|=o(1),
\end{array}
$$

and observe that $L_{2}$ (respectively $L_{3}$ ) has the same distribution as $L_{2}^{\prime}$ (respectively $L_{3}^{\prime}$ ).

Let us prove the first bound of (16). In view of (5) it suffices to show that $\tilde{d}_{T V}\left(L_{0}, L\right)=o_{P}(1)$. In order to prove the latter bound we apply the inequality

$$
\tilde{d}_{T V}\left(L_{0}, L\right) \mathbb{I}_{\mathcal{A}_{1}} \leqslant n^{-1} Y_{1}^{2} \hat{a}_{2}
$$


shown below. We remark that (18) implies

$$
\tilde{d}_{T V}\left(L_{0}, L\right) \leqslant \tilde{d}_{T V}\left(L_{0}, L\right) \mathbb{I}_{\mathcal{A}_{1}}+\mathbb{I}_{\overline{\mathcal{A}}_{1}} \leqslant n^{-1} Y_{1}^{2} \hat{a}_{2}+\mathbb{I}_{\overline{\mathcal{A}}_{1}}=o_{P}(1) .
$$

Here $n^{-1} Y_{1}^{2} \hat{a}_{2}=o_{P}(1)$, because $Y_{1}^{2} \hat{a}_{2}$ is stochastically bounded. Furthermore, the bound $\mathbb{I}_{\overline{\mathcal{A}}_{1}}=o_{P}(1)$ follows from (7). It remains to prove (18). We denote $\bar{L}_{k}=\sum_{l=1}^{k} \mathbb{I}_{l} u_{l}+$ $\sum_{l=k+1}^{m} \eta_{l} u_{l}$ and write, by the triangle inequality,

$$
\tilde{d}_{T V}\left(L_{0}, L\right) \leqslant \sum_{k=1}^{m} \tilde{d}_{T V}\left(\bar{L}_{k-1}, \bar{L}_{k}\right)
$$

Then we estimate $\tilde{d}_{T V}\left(\bar{L}_{k-1}, \bar{L}_{k}\right) \leqslant \tilde{d}_{T V}\left(\eta_{k}, \mathbb{I}_{k}\right) \leqslant(n m)^{-1} Y_{1}^{2} X_{k}^{2}$. Here the first inequality follows from the properties of the total variation distance. The second inequality follows from Lemma 6 and the fact that on the event $\mathcal{A}_{1}$ we have $p_{k 1}=\lambda_{k 1}$.

Let us prove the second bound of (16). In view of (5) it suffices to show that $\tilde{d}_{T V}\left(L_{0}, L_{1}\right)=o_{P}(1)$. We denote $L_{k}^{*}=\sum_{l=1}^{k} \eta_{l} u_{l}+\sum_{l=k+1}^{m} \eta_{l} \xi_{1 l}$ and write, by the triangle inequality,

$$
\tilde{d}_{T V}\left(L_{0}, L_{1}\right) \leqslant \sum_{k=1}^{m} \tilde{d}_{T V}\left(L_{k-1}^{*}, L_{k}^{*}\right)
$$

Here

$$
\tilde{d}_{T V}\left(L_{k-1}^{*}, L_{k}^{*}\right) \leqslant \tilde{d}_{T V}\left(\eta_{k} u_{k}, \eta_{k} \xi_{1 k}\right) \leqslant \tilde{\mathbf{P}}\left(\eta_{k} \neq 0\right) \tilde{d}_{T V}\left(u_{k}, \xi_{1 k}\right) .
$$

Now, invoking the inequalities $\tilde{\mathbf{P}}\left(\eta_{k} \neq 0\right)=1-e^{-\lambda_{k 1}} \leqslant \lambda_{k 1}$ and $\tilde{d}_{T V}\left(u_{k}, \xi_{1 k}\right) \leqslant \sum_{j=2}^{n} p_{k j}^{2}$, we obtain the desired bound from (8), (19) and (20)

$$
\tilde{d}_{T V}\left(L_{0}, L_{1}\right) \leqslant \sum_{k=1}^{m} \lambda_{k 1} \sum_{j=2}^{n} p_{k j}^{2} \leqslant Y_{1} S_{X Y}=o_{P}(1) .
$$

Let us prove the first bound of (17). We observe that

$$
\left|L_{2}-L_{1}\right|=L_{2}-L_{1}=\sum_{1 \leqslant k \leqslant m} \eta_{k} \Delta_{1 k}
$$

and

$$
\tilde{\mathbf{E}} \sum_{1 \leqslant k \leqslant m} \eta_{k} \Delta_{1 k}=\sum_{1 \leqslant k \leqslant m} \lambda_{k 1} \sum_{2 \leqslant j \leqslant n}\left(\lambda_{k j}-1\right) \mathbb{I}_{\left\{\lambda_{k j}>1\right\}} \leqslant Y_{1} S_{X Y} .
$$

Hence, we obtain $\mathbf{E}\left|L_{2}-L_{1}\right| \leqslant \mathbf{E} Y_{1} \mathbf{E} S_{X Y}=o(1)$, see (8).

Let us prove the second bound of (17). It suffices to show that $\tilde{\mathbf{E}}\left|L_{2}^{\prime}-L_{3}^{\prime}\right|=o_{P}(1)$. We have

$$
\tilde{\mathbf{E}}\left|L_{2}^{\prime}-L_{3}^{\prime}\right|=\left(\delta_{2}+\delta_{3}\right) Y_{1} \hat{a}_{2}=\left|\hat{b}_{1}-b_{1}\right| Y_{1} \hat{a}_{2}=o_{P}(1) .
$$

In the last step we used the fact that $Y_{1} \hat{a}_{2}=O_{P}(1)$ and $\hat{b}_{1}-b_{1}=o_{P}(1)$. 
The third bound of (17) is straightforward

$$
\mathbf{E}\left|L_{3}-L_{4}\right| \leqslant\left|\frac{1}{\sqrt{\beta_{n}}}-\frac{1}{\sqrt{\beta}}\right| b_{1} \sum_{1 \leqslant k \leqslant m} \mathbf{E} \eta_{k} X_{k}=\left|1-\frac{\sqrt{\beta_{n}}}{\sqrt{\beta}}\right| b_{1}^{2} a_{2}=o(1)
$$

Step 2. Here we show that for every real $t$ we have $\mathbf{E} \Delta(t)=o(1)$, where $\Delta(t)=$ $e^{i t L_{4}}-e^{i t d_{*}}$. To this aim we prove that for any realized value $Y_{1}$ there exists a positive constant $c=c\left(t, Y_{1}\right)$ such that for every $0<\varepsilon<0.5$ we have

$$
\limsup _{n, m \rightarrow+\infty}\left|\mathbf{E}\left(\Delta(t) \mid Y_{1}\right)\right|<c \varepsilon
$$

Clearly, (22) implies $\mathbf{E}\left(\Delta(t) \mid Y_{1}\right)=o(1)$. This fact together with the simple inequality $|\Delta(t)| \leqslant 2$ yields the desired bound $\mathbf{E} \Delta(t)=o(1)$, by Lebesgue's dominated convergence theorem.

We fix $0<\varepsilon<0.5$ and prove (22). Introduce event $\mathcal{D}=\left\{\left|\hat{a}_{1}-a_{1}\right|<\varepsilon \min \left\{1, a_{1}\right\}\right\}$ and let $\overline{\mathcal{D}}$ denote the complement event. Furthermore, select a number $T>1 / \varepsilon$ such that $\mathbf{P}\left(\tau_{1} \geqslant T\right)<\varepsilon$. By $c_{1}, c_{2}, \ldots$ we denote positive numbers which do not depend on $n, m$.

We observe that, given $Y_{1}$, the conditional distribution of $d_{*}$ is the compound Poisson distribution with the characteristic function $f(t)=e^{\lambda_{1}\left(f_{\tau}(t)-1\right)}$. Similarly, given $X, Y$ and $\xi_{41}, \ldots, \xi_{4 m}$, the conditional distribution of $L_{4}$ is the compound Poisson distribution with the characteristic function $\bar{f}(t)=e^{\bar{\lambda}\left(\bar{f}_{\tau}(t)-1\right)}$. In the proof of (22) we exploit the convergence $\bar{\lambda} \rightarrow \lambda_{1}$ and $\bar{f}_{\tau}(t) \rightarrow f_{\tau}(t)$. In what follows we assume that $m, n$ are so large that $\beta \leqslant 2 \beta_{n} \leqslant 4 \beta$.

Let us show (22). For this purpose we write

$$
\mathbf{E}\left(\Delta(t) \mid Y_{1}\right)=\mathbf{E}_{1} \Delta(t)=I_{1}+I_{2}, \quad I_{1}=\mathbf{E}_{1} \Delta(t) \mathbb{I}_{\mathcal{D}}, \quad I_{2}=\mathbf{E}_{1} \Delta(t) \mathbb{I}_{\overline{\mathcal{D}}}
$$

and estimate $I_{1}$ and $I_{2}$. We have $\left|I_{2}\right| \leqslant 2 \mathbf{P}_{1}(\overline{\mathcal{D}})=2 \mathbf{P}(\overline{\mathcal{D}})=o(1)$, by the law of large numbers.

Now we show that $I_{1} \leqslant c \varepsilon+o(1)$. Combining the identity $\mathbf{E}_{1} \Delta(t)=\mathbf{E}_{1} f(t)\left(e^{\delta}-1\right)$ with the inequalities $|f(t)| \leqslant 1$ and $\left|e^{s}-1\right| \leqslant|s| e^{|s|}$ we obtain

$$
\left|I_{1}\right| \leqslant \mathbf{E}_{1}|\delta| e^{|\delta|} \mathbb{I}_{\mathcal{D}} \leqslant c_{1} \mathbf{E}_{1}|\delta| \mathbb{I}_{\mathcal{D}}
$$

Here we estimated $e^{|\delta|} \leqslant e^{8 \lambda_{1}}=: c_{1}$ using the inequalities

$$
|\delta| \leqslant 2 \bar{\lambda}+2 \lambda_{1}, \quad \bar{\lambda}=Y_{1} \beta_{n}^{1 / 2} \hat{a}_{1} \leqslant 3 \lambda_{1} .
$$

We remark that the last inequality holds for $\beta_{n} \leqslant 2 \beta$ provided that the event $\mathcal{D}$ occurs. We shall show below that

$$
\mathbf{E}_{1}|\delta| \mathbb{I}_{\mathcal{D}} \leqslant\left(c_{2}+\lambda_{1} c_{3}+\lambda_{1} c_{4}\right) \varepsilon+o(1)
$$

This inequality together with (23) yields the desired upper bound for $I_{1}$. 
Let us prove (24). We write

$$
\delta=\left(\bar{f}_{\tau}(t)-1\right)\left(\bar{\lambda}-\lambda_{1}\right)+\left(\bar{f}_{\tau}(t)-f_{\tau}(t)\right) \lambda_{1}
$$

and estimate

$$
|\delta| \leqslant 2\left|\bar{\lambda}-\lambda_{1}\right|+\lambda_{1}\left|\bar{f}_{\tau}(t)-f_{\tau}(t)\right| \text {. }
$$

Now, from the inequalities $\left|\hat{a}_{1}-a_{1}\right|<\varepsilon$ and $\beta_{n} \leqslant 2 \beta$ we obtain

$$
\left|\bar{\lambda}-\lambda_{1}\right| \leqslant Y_{1}\left|\hat{a}_{1}-a\right| \beta_{n}^{1 / 2}+Y_{1} a_{1}\left|\beta_{n}^{1 / 2}-\beta^{1 / 2}\right| \leqslant 2 Y_{1} \beta^{1 / 2} \varepsilon+o(1) .
$$

Hence, $\mathbf{E}_{1}\left|\bar{\lambda}-\lambda_{1}\right| \mathbb{I}_{\mathcal{D}} \leqslant c_{2} \varepsilon+o(1)$, where $c_{2}=2 Y_{1} \beta^{1 / 2}$. We complete the proof of (24) by showing that

$$
\mathbf{E}_{1}\left|\bar{f}_{\tau}(t)-f_{\tau}(t)\right| \mathbb{I}_{\mathcal{D}} \leqslant\left(c_{3}+c_{4}\right) \varepsilon+o(1) .
$$

In order to prove (25) we split

$$
\bar{f}_{\tau}(t)-f_{\tau}(t)=\sum_{r \geqslant 0} e^{i t r}\left(\bar{p}_{r}-p_{r}\right)=R_{1}-R_{2}+R_{3},
$$

and estimate separately the terms

$$
R_{1}=\sum_{r \geqslant T} e^{i t r} \bar{p}_{r}, \quad R_{2}=\sum_{r \geqslant T} e^{i t r} p_{r}, \quad R_{3}=\sum_{0 \leqslant r<T} e^{i t r}\left(\bar{p}_{r}-p_{r}\right) .
$$

Here we denote $p_{r}=\mathbf{P}\left(\tau_{1}=r\right)$. The upper bound for $R_{2}$ follows by the choice of $T$

$$
\left|R_{2}\right| \leqslant \sum_{r \geqslant T} p_{r}=\mathbf{P}\left(\tau_{1} \geqslant T\right)<\varepsilon .
$$

Next, combining the identity $\bar{p}_{r}=\left(\hat{a}_{1} m\right)^{-1} \sum_{1 \leqslant k \leqslant m} X_{k} \mathbb{I}_{\left\{\xi_{4 k}=r\right\}}$ with the inequalities

$$
\left|R_{1}\right| \leqslant\left(\hat{a}_{1} m\right)^{-1} \sum_{r \geqslant T} \sum_{1 \leqslant k \leqslant m} X_{k} \mathbb{I}_{\left\{\xi_{4 k}=r\right\}}=\left(\hat{a}_{1} m\right)^{-1} \sum_{1 \leqslant k \leqslant m} X_{k} \mathbb{I}_{\left\{\xi_{4 k} \geqslant T\right\}}
$$

and $\hat{a}_{1}^{-1} \mathbb{I}_{\mathcal{D}} \leqslant 2 a_{1}^{-1}$, we estimate

$$
\mathbf{E}_{1}\left|R_{1}\right| \mathbb{I}_{\mathcal{D}} \leqslant 2 a_{1}^{-1} \mathbf{E}_{1}\left(X_{k} \mathbb{I}_{\left\{\xi_{4 k} \geqslant T\right\}}\right) \leqslant 2 a_{1}^{-1} T^{-1} \mathbf{E}_{1}\left(X_{k} \xi_{4 k}\right)=2 a_{1}^{-1} a_{2} b_{1} \beta^{-1 / 2} \varepsilon .
$$

Hence, we obtain $\mathbf{E}_{1}\left|R_{1}\right| \mathbb{I}_{\mathcal{D}} \leqslant c_{4} \varepsilon$, where $c_{4}=2 a_{1}^{-1} a_{2} b_{1} \beta^{-1 / 2}$.

Now we estimate $R_{3}$. We denote $p_{r}^{\prime}=\left(\hat{a}_{1} / a_{1}\right) \bar{p}_{r}$ and observe that the inequality $\left|\hat{a}_{1}-a\right| \leqslant \varepsilon a_{1}$ implies $\left|\hat{a}_{1} a_{1}^{-1}-1\right| \leqslant \varepsilon$ and

$$
\left|\sum_{0 \leqslant r \leqslant T} e^{i t r}\left(\bar{p}_{r}-p_{r}^{\prime}\right)\right| \leqslant \varepsilon \sum_{0 \leqslant r \leqslant T} \bar{p}_{r} \leqslant \varepsilon .
$$

In the last inequality we use the fact that the probabilities $\left\{\bar{p}_{r}\right\}_{r \geqslant 0}$ sum up to 1 . It follows now that

$$
\left|R_{3}\right| \mathbb{I}_{\mathcal{D}} \leqslant \varepsilon+\sum_{0 \leqslant r \leqslant T}\left|p_{r}^{\prime}-p_{r}\right|
$$


Furthermore, observing that $\mathbf{E}_{1} p_{r}^{\prime}=a^{-1} \mathbf{E}_{1} X_{k} \mathbb{I}_{\left\{\xi_{4 k}=r\right\}}=p_{r}$, for $1 \leqslant k \leqslant m$, we obtain

$$
\mathbf{E}_{1}\left|p_{r}^{\prime}-p_{r}\right|^{2}=m^{-1} \mathbf{E}_{1}\left|a^{-1} X_{k} \mathbb{I}_{\left\{\xi_{4 k}=r\right\}}-p_{r}\right|^{2} \leqslant m^{-1} a_{1}^{-2} \mathbf{E} X_{k}^{2} .
$$

Hence, $\mathbf{E}_{1}\left|p_{r}^{\prime}-p_{r}\right|=O\left(m^{-1 / 2}\right)$. We conclude that

$$
\mathbf{E}_{1}\left|R_{3}\right| \mathbb{I}_{\mathcal{D}} \leqslant \varepsilon+O\left(|T| m^{-1 / 2}\right)=\varepsilon+o(1),
$$

thus completing the proof of (25).

The case (iii). Before the proof we introduce several random variables. Given $\varepsilon \in$ $(0,1)$, denote

$$
\mathbb{I}_{k}^{\prime}=\mathbb{I}_{\left\{X_{k} \beta_{n}^{-1 / 2} b_{1}<\varepsilon\right\}}, \quad \gamma_{k}=X_{k} \beta_{n}^{-1 / 2} b_{1} \mathbb{I}_{k}^{\prime}, \quad 1 \leqslant k \leqslant m, \quad \text { and } \quad \gamma=\sum_{1 \leqslant k \leqslant m} \lambda_{k 1} \gamma_{k} .
$$

Given $X, Y$, let $\tilde{\mathbb{I}}_{1}, \ldots, \tilde{\mathbb{I}}_{m}$ be conditionally independent Bernoulli random variables with success probabilities

$$
\tilde{\mathbf{P}}\left(\tilde{\mathbb{I}}_{k}=1\right)=1-\tilde{\mathbf{P}}\left(\tilde{\mathbb{I}}_{k}=0\right)=\gamma_{k} .
$$

We assume that, given $X, Y$, the sequences $\left\{\mathbb{I}_{k}\right\}_{k=1}^{m},\left\{\tilde{\mathbb{I}}_{k}\right\}_{k=1}^{m}$ and $\left\{\xi_{3 k}\right\}_{k=1}^{m}$ (see (15)) are conditionally independent. Furthermore, introduce random variables

$$
L_{5}=\sum_{1 \leqslant k \leqslant m} \mathbb{I}_{k} \xi_{3 k}, \quad L_{6}=\sum_{1 \leqslant k \leqslant m} \mathbb{I}_{k} \mathbb{I}_{k}^{\prime} \xi_{3 k}, \quad L_{7}=\sum_{1 \leqslant k \leqslant m} \mathbb{I}_{k} \tilde{\mathbb{I}}_{k}
$$

and let $L_{8}$ be a random variable with the distribution $\mathbf{P}\left(L_{8}=r\right)=\mathbf{E} e^{-\gamma} \gamma^{r} / r$ !, for $r=0,1, \ldots$ We remark that the probability distribution of $L_{8}$ is a Poisson mixture, i.e., the Poisson distribution with random parameter $\gamma$.

We note that by (16) and the first two bounds of (17), the random variables $L$ and $L_{3}$ have the same asymptotic distribution (if any). Now we prove that $L_{3}$ converges in distribution to $\Lambda_{3}$. For this purpose we show that for any $\varepsilon \in(0,1)$

$$
\begin{aligned}
& d_{T V}\left(L_{3}, L_{5}\right)=o(1), \quad \mathbf{E}\left|L_{5}-L_{6}\right|=o(1), \\
& d_{T V}\left(L_{6}, L_{7}\right) \leqslant a_{2} b_{1}^{2} \varepsilon, \quad d_{T V}\left(L_{7}, L_{8}\right)=o(1), \\
& \mathbf{E} e^{i t L_{8}}-\mathbf{E} e^{i t \Lambda_{3}}=o(1) .
\end{aligned}
$$

Let us prove (27). The first bound of (27) is obtained in the same way as the first bound of (16). To show the second bound of (27) we write

$$
\tilde{\mathbf{E}}\left|L_{5}-L_{6}\right|=\sum_{1 \leqslant k \leqslant m}\left(1-\mathbb{I}_{k}^{\prime}\right) \tilde{\mathbf{E}}_{k} \tilde{\mathbf{E}} \xi_{3 k} \leqslant Y_{1} b_{1} m^{-1} \sum_{1 \leqslant k \leqslant m}\left(1-\mathbb{I}_{k}^{\prime}\right) X_{k}^{2}
$$

and obtain

$$
\mathbf{E}\left|L_{5}-L_{6}\right|=\mathbf{E} \tilde{\mathbf{E}}\left|L_{5}-L_{6}\right| \leqslant b_{1}^{2} \mathbf{E} X_{1}^{2} \mathbb{I}_{\left\{X_{1} \beta_{n}^{-1 / 2} b_{1} \geqslant \varepsilon\right\}}=o(1) .
$$

We note that the right hand side tends to zero since $\beta_{n} \rightarrow+\infty$. 
Let us prove the first inequality of (28). Proceeding as in (19), (20) and using the identity $\tilde{\mathbb{I}}_{k}=\tilde{\mathbb{I}}_{k} \mathbb{I}_{k}^{\prime}$ we write

$$
\tilde{d}_{T V}\left(L_{6}, L_{7}\right) \leqslant \sum_{1 \leqslant k \leqslant m} \mathbb{I}_{k}^{\prime} \tilde{\mathbf{P}}\left(\mathbb{I}_{k} \neq 0\right) \tilde{d}_{T V}\left(\xi_{3 k}, \tilde{\mathbb{I}}_{k}\right)
$$

Next, we estimate $\mathbb{I}_{k}^{\prime} \tilde{d}_{T V}\left(\xi_{3 k}, \tilde{\mathbb{I}}_{k}\right) \leqslant \gamma_{k}^{2}$, by LeCam's inequality (14), and invoke the inequality $\tilde{\mathbf{P}}\left(\mathbb{I}_{k} \neq 0\right) \leqslant \lambda_{k 1}$. We obtain

$$
\tilde{d}_{T V}\left(L_{6}, L_{7}\right) \leqslant \sum_{1 \leqslant k \leqslant m} \mathbb{I}_{k}^{\prime} \lambda_{k 1} \gamma_{k}^{2} \leqslant \varepsilon \sum_{1 \leqslant k \leqslant m} \mathbb{I}_{k}^{\prime} \lambda_{k 1} \gamma_{k} \leqslant \varepsilon Y_{1} b_{1} \hat{a}_{2}
$$

Here we estimated $\gamma_{k}^{2} \leqslant \varepsilon \gamma_{k}$. Now the inequalities $d_{T V}\left(L_{6}, L_{7}\right) \leqslant \mathbf{E} \tilde{d}_{T V}\left(L_{6}, L_{7}\right) \leqslant a_{2} b_{1}^{2} \varepsilon$ imply the first relation of (28).

Let us prove the second relation of (28). In view of (5) it suffices to show that $\tilde{d}_{T V}\left(L_{7}, L_{8}\right)=o_{P}(1)$. For this purpose we write

$$
\tilde{d}_{T V}\left(L_{7}, L_{8}\right) \leqslant \mathbb{I}_{\mathcal{A}_{1}} \tilde{d}_{T V}\left(L_{7}, L_{8}\right)+\mathbb{I}_{\overline{\mathcal{A}}_{1}},
$$

where $\mathbb{I}_{\overline{\mathcal{A}}_{1}}=o_{P}(1)$, see $(7)$, and estimate using LeCam's inequality (14)

$$
\mathbb{I}_{\mathcal{A}_{1}} \tilde{d}_{T V}\left(L_{7}, L_{8}\right) \leqslant \mathbb{I}_{\mathcal{A}_{1}} \sum_{1 \leqslant k \leqslant m} \tilde{\mathbf{P}}^{2}\left(\mathbb{I}_{k} \tilde{\mathbb{I}}_{k}=1\right) \mathbb{I}_{k}^{\prime}=\mathbb{I}_{\mathcal{A}_{1}} \sum_{1 \leqslant k \leqslant m} \lambda_{k 1}^{2} \gamma_{k}^{2} \leqslant b_{1}^{2} Y_{1}^{2} m^{-1} \hat{a}_{4}=o_{P}(1) .
$$

In the last step we used the fact that $\mathbf{E} X_{1}^{2}<\infty$ implies $m^{-1} \hat{a}_{4}=o_{P}(1)$.

Finally, we show (29). We write $\tilde{\mathbf{E}} e^{i t L_{8}}=e^{\gamma\left(e^{i t}-1\right)}$ and observe that

$$
Y_{1} b_{1} a_{2}-\gamma=o_{P}(1)
$$

Furthermore, since for any real $t$ the function $z \rightarrow e^{z\left(e^{i t}-1\right)}$ is bounded and uniformly continuous for $z \geqslant 0$, we conclude that (30) implies the convergence

$$
\mathbf{E} e^{i t L_{8}}=\mathbf{E} e^{\gamma\left(e^{i t}-1\right)} \rightarrow \mathbf{E} e^{Y_{1} b_{1} a_{2}\left(e^{i t}-1\right)}=\mathbf{E} e^{i t \Lambda_{3}} .
$$

It remains to prove (30). We write $Y_{1} b_{1} a_{2}-\gamma=Y_{1} b_{1}\left(a_{2}-\hat{a}_{2}\right)+Y_{1} b_{1} \hat{a}_{2}-\gamma$ and note that $a_{2}-\hat{a}_{2}=o_{P}(1)$, by the law of large numbers, and

$$
0 \leqslant \mathbf{E}\left(Y_{1} b_{1} \hat{a}_{2}-\gamma\right)=b_{1}^{2} \mathbf{E} X_{1}^{2} \mathbb{I}_{\left\{X_{1} \beta_{n}^{-1 / 2} b_{1} \geqslant \varepsilon\right\}}=o(1) .
$$

\section{Acknowledgements}

We thank the anonymous referee for remarks and suggestions that have improved the presentation. 


\section{References}

[1] A. D. Barbour and G. Reinert. The shortest distance in random multi-type intersection graphs. Random Structures and Algorithms, 39:179-209, 2011.

[2] M. Behrisch. Component evolution in random intersection graphs. Electron. J. Combin., 14:Research Paper 17, 12, 2007.

[3] S. Blackburn and S. Gerke. Connectivity of the uniform random intersection graph. Discrete Mathematics, 309: 5130-5140, 2009.

[4] M. Bloznelis. Degree distribution of a typical vertex in a general random intersection graph. Lithuanian Mathematical J., 48:38-45, 2008.

[5] M. Bloznelis. A random intersection digraph: Indegree and outdegree distributions. Discrete Mathematics, 310:2560-2566, 2010.

[6] M. Bloznelis. The largest component in an inhomogeneous random intersection graph with clustering. Electron. J. Combin., 17:Research Paper 110, 17, 2010.

[7] M. Bloznelis. Degree and clustering coefficient in sparse random intersection graphs. Annals of Applied Probability, 23:1254-1289, 2013.

[8] M. Bloznelis, V. Kurauskas. Clustering function: a measure of social influence. arXiv: 1207.4941, 2012

[9] M. Bradonjic, A. Hagberg, N. W. Hengartner, and A. G. Percus. Component Evolution in General Random Intersection Graphs. In The 7th Workshop on Algorithms and Models for the Web Graph, WAW2010, volume 6516 of Lecture Notes in Comput. Sci., pages 36-49. Springer, 2010.

[10] T. Britton, M. Deijfen, M. Lindholm, and N. A. Lageras. Epidemics on random graphs with tunable clustering. J. Appl. Prob., 45:743-756, 2008.

[11] M. Deijfen and W. Kets. Random intersection graphs with tunable degree distribution and clustering. Probab. Engrg. Inform. Sci., 23:661-674, 2009.

[12] L. Eschenauer and V. D. Gligor. A key-management scheme for distributed sensor networks. In Proceedings of the 9th ACM Conference on Computer and Communications Security, pages 41-47. ACM New York, 2002.

[13] S. Foss, D. Korshunov, and S. Zachary. An Introduction to Heavy-Tailed and Subexponential Distributions. ACM, New York, 2011.

[14] E. Godehardt and J. Jaworski. Two models of random intersection graphs and their applications. Electronic Notes in Discrete Mathematics, 10:129-132, 2001.

[15] J. Jaworski, M. Karoński, and D. Stark. The degree of a typical vertex in generalized random intersection graph models. Discrete Mathematics, 306:2152-2165, 2006.

[16] J. Jaworski and D. Stark. The vertex degree distribution of passive random intersection graph models. Combinatorics, Probability and Computing, 17:549-558, 2008.

[17] M. Karoński, E. R. Scheinerman, and K. B. Singer-Cohen. On random intersection graphs: The subgraph problem. Combinatorics, Probability and Computing, 8:131159, 1999. 
[18] S. Nikoletseas, C. Raptopoulos, and P. G. Spirakis. The existence and efficient construction of large independent sets in general random intersection graphs. In ICALP (2004), J. Daz, J. Karhumki, A. Lepist, and D. Sannella, Eds., volume 3142 of Lecture Notes in Comput. Sci., pages 1029-1040. Springer, 2004.

[19] K. Rybarczyk. Diameter, connectivity, and phase transition of the uniform random intersection graph. Discrete Mathematics, 311:1998-2019, 2011.

[20] K. Rybarczyk. The degree distribution in random intersection graphs. In Studies in Classification, Data Analysis and Knowledge Organization, pages 291-299. Springer, 2012.

[21] Y. Shang. Degree distributions in general random intersection graphs. Electron. J. Combin., 17:Research Paper 23, 8, 2010.

[22] K. B. Singer-Cohen. Random intersection graphs. PhD thesis, Department of Mathematical Sciences, The Johns Hopkins University, 1995.

[23] D. Stark. The vertex degree distribution of random intersection graphs. Random Structures and Algorithms, 24:249-258, 2004.

[24] J. M. Steele. Le Cam's inequality and Poisson approximations. The American Mathematical Monthly, 101:48-54, 1994. 